\title{
Leptomeningeal disease in oligodendroglial tumors: a population-based study
}

\author{
Gloria Roldán · Jennifer Chan • Misha Eliasziw • \\ J. Gregory Cairncross $\cdot$ Peter A. Forsyth
}

Received: 6 January 2011/Accepted: 18 February 2011/Published online: 4 March 2011

(C) The Author(s) 2011. This article is published with open access at Springerlink.com

\begin{abstract}
In this population-based study, we determined the frequency and clinical characteristics of leptomeningeal disease (LMD) developing in the context of oligodendroglial tumors (oligodendrogliomas and oligoastrocytomas). LMD occurred in only $3.9 \%(8 / 204)$ of oligodendroglial tumors and in patients with more recurrences [mean 2.88 vs. 1.27 in LMD and non-LMD, respectively $(p=0.001)]$. In contrast to LMD from systemic solid tumors, the median survival following the diagnosis of LMD in oligodendroglial tumors was surprisingly long at 22 months (95\% CI 11-33 months). Treatment with oral chemotherapy seemed as effective as more aggressive treatments (e.g. repeat RT or intrathecal chemotherapy) in these patients.
\end{abstract}

G. Roldán · J. Chan · J. G. Cairncross · P. A. Forsyth (凹)

Clark H. Smith Brain Tumor Center, Tom Baker Cancer Center, Alberta Health Services, HRIC 2AA07, 3330 Hospital Dr NW, Calgary, AB T2N 4N1, Canada

e-mail: pforsyth@ucalgary.ca

G. Roldán · P. A. Forsyth

Department of Oncology, University of Calgary, 1331 29th

Street NW, Calgary, AB T2N 4N2, Canada

J. Chan

Department of Pathology and Laboratory Medicine, University of Calgary, 1331 29th Street NW, Calgary, AB T2N 4N2, Canada

M. Eliasziw

Department of Community Health Sciences, University of Calgary, 1331 29th Street NW, Calgary, AB T2N 4N2, Canada

J. G. Cairncross · P. A. Forsyth

Department of Clinical Neurosciences, University of Calgary,

1403 29th Street NW, Calgary, AB T2N 2T9, Canada
Keywords Oligodendroglial tumors · Leptomeningeal spread

\section{Introduction}

Oligodendroglial tumors (oligodendrogliomas and mixed oligoastrocytomas) represent $5-20 \%$ of gliomas [1, 2]. In oligodendroglial tumors, co-deletion of chromosomes $1 \mathrm{p}$ and $19 q$ (likely a manifestation of a recurring unbalanced $1 \mathrm{q} / 19 \mathrm{p}$ translocation) is associated with better overall prognosis [3-5] and response to treatment [6-8]. We recently encountered a series of patients with the unusual complication of leptomeningeal disease (LMD) from oligodendroglial tumors which had several unusual features (e.g. survival with LMD of up to 3.6 years in one patient) [9]. We performed this study to determine how commonly this occurred at the population level and to identify clinical features that might predict the occurrence of LMD.

\section{Patients and methods}

Ethical approval was received from the Conjoint Health Research Ethics Board of the University of Calgary to review charts for every patient diagnosed with an oligodendroglial tumor (oligodendrogliomas and mixed oligoastrocytomas WHO grades II and III) diagnosed in Southern Alberta (population 1.8 million) between 1 July 1991 and 31 Aug 2009. Patients were identified through the Alberta Cancer Registry and their individual records were reviewed.

Of the 208 identified patients, four were excluded because they were lost to follow-up without data available. In our institution $1 \mathrm{p} / 19 \mathrm{q}$ status was evaluated in the 
primary tumor by either polymerase chain reaction (PCR) or fluorescent in situ hybridization (FISH) techniques.

The criteria for the diagnosis of leptomeningeal disease (LMD) were any two or more of the following [9]: (1) neurological symptoms or signs consistent with LMD, (2) MRI changes consistent with LMD (i.e., leptomeningeal, subependymal, or subarachnoid spread), or (3) a cytological diagnosis of LMD on examination of cerebrospinal fluid (CSF).

\section{Statistics}

Results were analyzed with SPSS Version 16.0 for Microsoft Windows and were two-tailed. For continuous variables, Spearman's rank correlation test was used to identify associations. For categorical variables, Chi-square or Fisher's exact tests were used. When comparing the means of continuous variables between groups, an unpaired two group $t$-test was used.

Progression-free survival (PFS) was defined as the interval between initial diagnosis and evidence of recurrence/progression, death, or lost to follow up. Overall survival (OS) was calculated from date of initial diagnosis to death or lost to follow-up. Time to first onset of LMD was calculated from initial tumor diagnosis.

The Kaplan-Meier method was used to estimate distributions for PFS and OS. The log-rank test was used to assess differences between these distributions with respect to the occurrence of clinical variables. Cox multivariate analysis (forward stepwise Wald) was done to assess their independent predictive value. All tests were two-tailed and $p$-values less than 0.05 were considered statistically significant.

\section{Results}

Population-based analysis of oligodendroglial tumors and risk of LMD

From July 1991 to August 2009, 208 patients in Southern Alberta were diagnosed with oligodendroglial grade II and III tumors; 204 were included in this analysis. This corresponds to an incidence of 1/100,000/year. Their characteristics are summarized in Table 1. For the entire group,

Table 1 Clinical characteristics of patients with oligodendroglial tumors that developed leptomeningeal disease compared with 196 that did not

\begin{tabular}{|c|c|c|c|c|}
\hline & $\begin{array}{l}\text { Total Population } \\
(n=204)\end{array}$ & $\begin{array}{l}\text { LMD } \\
(n=8)^{\mathrm{a}}\end{array}$ & $\begin{array}{l}\text { No LMD } \\
(n=196)^{\mathrm{b}}\end{array}$ & $\begin{array}{l}\text { Significance } \\
(p)^{\mathrm{a}} \text { vs. }{ }^{\mathrm{b}}\end{array}$ \\
\hline Mean age in years (range) & $42(18-78)$ & $40(28-50)$ & $42(18-78)$ & 0.636 \\
\hline Median KPS (range) & $85(50-100)$ & $85(70-90)$ & $90(50-100)$ & 0.477 \\
\hline Debulking (\%) & $173(85)$ & $7(87.5)$ & $166(85)$ & 0.828 \\
\hline Pathology at diagnosis $(\%)$ & & & & 0.079 \\
\hline - Oligo. Gr. II & $62(30.5)$ & $1(12.5)$ & $61(31.1)$ & \\
\hline - Oligo. Gr. III & 49 (24) & $5(62.5)$ & $44(22.4)$ & \\
\hline - Mixed OA Gr. II & $52(25.5)$ & $1(12.5)$ & $51(26)$ & \\
\hline - Mixed OA Gr. III & $41(20)$ & $1(12.5)$ & $40(20.4)$ & \\
\hline $1 \mathrm{p} / 19 \mathrm{q}$ & & & & 0.127 \\
\hline - Not tested (\%) & $43(21)$ & $1 / 7(14)$ & $42(21)$ & \\
\hline - Codeleted ${ }^{\mathrm{c}}(\%$ of tested $)$ & $93 / 161(58)$ & $6 / 7(86)$ & $87 / 154(56.4)$ & \\
\hline Initial treatment $(\%)$ & & & & 0.785 \\
\hline - Observation & $96(47)$ & $5(62.5)$ & $91(46)$ & \\
\hline - RT alone & $35(17)$ & $1(12.5)$ & $34(17.3)$ & \\
\hline - ChT alone & $18(9)$ & $1(12.5)$ & $17(9)$ & \\
\hline - SeqRTChT & $26(13)$ & 0 & $26(13.3)$ & \\
\hline - ConcRTChT & $29(14)$ & $1(12.5)$ & $28(14.3)$ & \\
\hline Mean number of recurrences & 1.35 & 2.88 & 1.27 & 0.001 \\
\hline PFS (months) (mean; 95\% CI) & $67(59-75)$ & $47(22-72)$ & $68(59-77)$ & 0.176 \\
\hline OS (months) (mean; 95\% CI) & $130(118-142)$ & $107(82-132)$ & $133(120-146)$ & 0.063 \\
\hline
\end{tabular}

LMD leptomeningeal disease, Oligo oligodendroglioma, $O A$ oligoastrocytoma, $R T$ radiotherapy, ChT chemotherapy, SeqRTChT sequential RT followed by ChT, ConcRTChT concurrent RT and ChT, PFS progression free survival, OS overall Survival

${ }^{a}$ Patients with leptomeningeal disease

b Patients without leptomeningeal disease

${ }^{c}$ Includes patients with only $1 \mathrm{p}$ loss 
mean age at diagnosis was 42 years (range 18-78) and 120 patients (59\%) were male. None of the 204 patients had oligodendroglial tumors originated from the posterior fossa. Four patients had bifrontal tumors and the rest were distributed homogeneously in the hemispheres with 108/200 $(54 \%)$ in the left hemisphere. 137/204 (67\%) of the patients had tumors involving the frontal lobes. Biomarker data was available on 161 patients (79\%); 93 (58\%) were co-deleted for $1 \mathrm{p}$ and $19 \mathrm{q}$.

With respect to initial treatment, 85/114 (75\%) patients with low grade tumors were observed (i.e., received no tumor treatment beyond surgery) while 78/90 (87\%) with anaplastic tumors received immediate postoperative treatment that consisted of radiotherapy (RT) alone (14\%), chemotherapy (ChT) alone (24\%), RT followed by ChT (32\%) or concurrent RT and ChT (30\%).

We analyzed clinical factors associated with OS and found that published prognostic factors were relevant in our population. Univariate analysis for OS showed that females lived longer (Log Rank test; $p=0.036$ ). Among grade III tumors, patients with pure oligodendrogliomas lived a mean of 124 months compared to 84 months for those with mixed tumors $(p<0.0001)$. Patients with WHO grade II histology lived longer than those with anaplastic (grade III) tumors $(p<0.0001)$ and co-deletion of $1 \mathrm{p} / 19 \mathrm{q}$ was associated with better overall survival $(p=0.004)$. Extent of initial resection did not impact OS (even when controlling for pathology, WHO grade, or genetic subtype). Tumors recurred in 122 patients and 73 had further surgery. Surgery at first recurrence was associated with longer OS (mean OS of 140 vs. 97 months; $p=0.001$ ).

Comparison of clinical and pathological characteristics in patients with LMD

Only $3.9 \%$ (8 of 204) of patients with oligodendroglial tumors developed LMD during the 18 year study period (Table 1). The only factor statistically associated with LMD was the number of tumor recurrences that were higher in the LMD group (mean of 2.88 recurrences) as compared to the no-LMD group (1.27 recurrences; $p=0.001$ ). There was no significant difference in patients with or without LMD in terms of age, KPS, surgical resection, pathology [pure vs. mixed; $p=0.47$ ) or low grade vs. high grade $(p=0.073)$ ], co-deletion of $1 \mathrm{p} / 19 \mathrm{q}$ $(p=0.127)$, or initial postoperative treatment $(p=0.785)$.

\section{Outcomes in patients with LMD}

The mean time from the initial diagnosis of a brain tumor until the diagnosis of LMD was 77 months $(95 \%$ CI 51-102 months). Surprisingly, in contrast to LMD from systemic solid tumors or GBM which have a universally
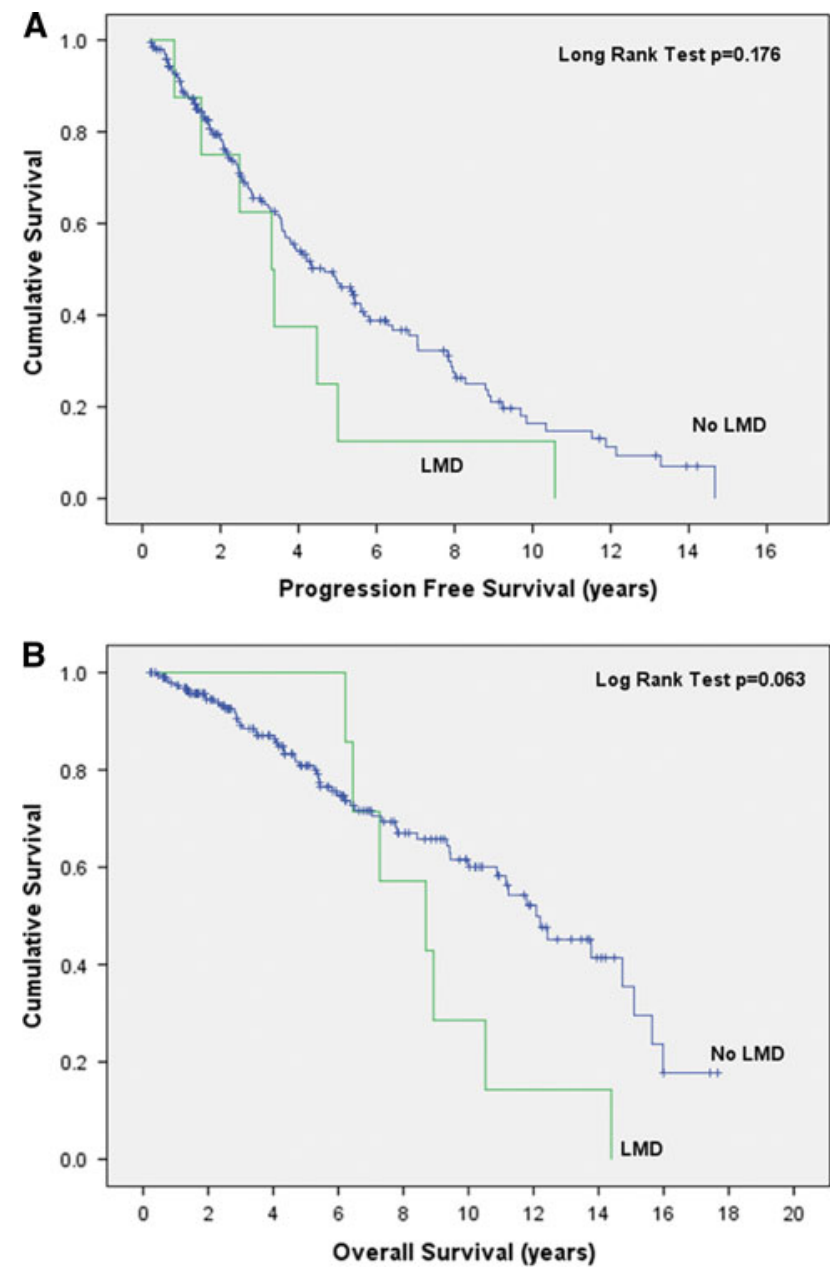

Fig. 1 Progression free survival (a) and overall survival (b) of patients with oligodendroglial tumors with and without LMD measured from the initial pathological diagnosis

poor prognosis, the progression-free and overall survivals were not statistically different between patients with and without LMD (Table 1) although there was a trend for both to be shorter in patients with LMD (Fig. 1). The mean survival following the diagnosis of LMD mean was 22 months (95\% CI 11-33 months) but this varied considerably from 1.8 to 42 months (Fig. 2).

\section{Subanalysis in patients with $1 \mathrm{p} / 19 \mathrm{q}$ co-deletion}

Considering the subjectivity and evolution of pathologic criteria for the diagnosis of pure and mixed oligodendroglial tumors, we thought that genetic subtyping would provide a more objective classification. We included a total of 93 patients that had $1 \mathrm{p} / 19 \mathrm{q}$ co-deletion or $1 \mathrm{p}$ loss. In this subgroup again the number of recurrences was associated with development of LMD with a mean of 1.1 and 2.8 recurrences for patients without and with LMD, respectively $(p=0.004) .0 .2 \%(1 / 53)$ and $12.5 \%(5 / 40)$ of patients with low and high grade tumors developed LMD, 


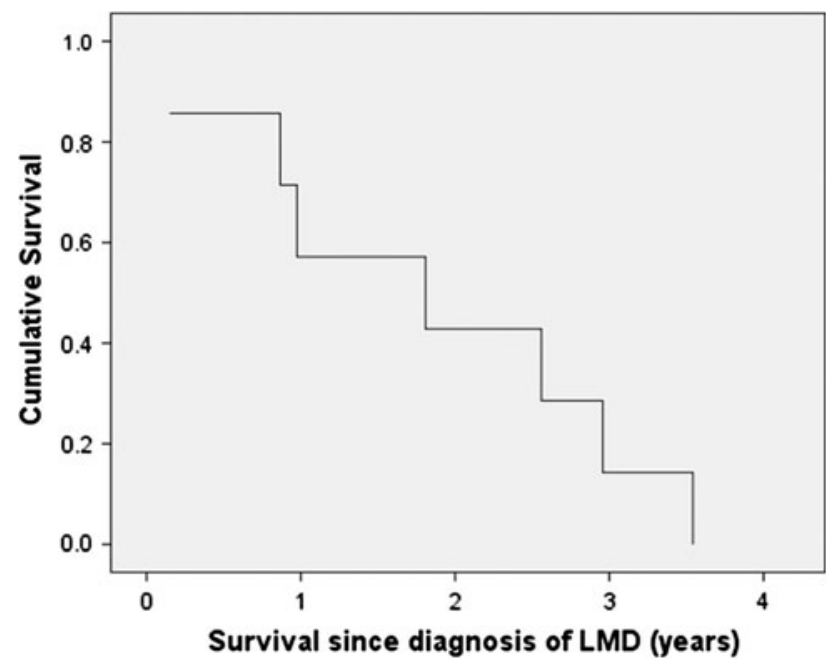

Fig. 2 Overall survival of 8 patients with oligodendroglial tumors from diagnosis of LMD to death

respectively $(p=0.04)$. In this subgroup the median survival was 107 months (95\% CI 101-113 months) for patients that developed LMD. This was significantly shorter than 177 months (95\% CI 155-199 months) for patients without $\operatorname{LMD}(p=0.033)$.

\section{Discussion}

To our knowledge, this is the first population-based study of the rare complication of LMD in oligodendroglial tumors. Other population-based studies of oligodendroglioma [10, 11] have not reported this complication. We found that LMD occurred in $3.9 \%$ of patients with oligodendroglial tumors, usually later in the natural history of the disease. The only clinical factor statistically associated with its development was the number of tumor recurrences. This could also be a surrogate for multiple surgeries but incomplete information on treatment of recurrences precludes a definitive conclusion. There was a trend for LMD to develop in patients with anaplastic tumors with $1 \mathrm{p} 19 \mathrm{q}$ co-deletion and in those who were observed initially rather than treated with chemotherapy or radiotherapy immediately after diagnosis. In patients harboring $1 \mathrm{p} / 19 \mathrm{q}$ codeletion, the diagnoses of LMD implied shorter OS. Survival following the diagnosis of LMD varied widely but on average was almost 2 years. This contrasts with rapidly fatal LMD in those with GBM or systemic solid tumors [12, 13].

Our subgroup analysis of anaplastic oligodendrogliomas found a trend for patients who did not receive postoperative treatment with radio- or chemotherapy to develop LMD.

We are uncertain about optimal treatment in this setting. Our patients tolerated various therapies, including intrathecal thiotepa, but we found no complete or partial responses. We now avoid potentially toxic therapies in these patients with longer survivals and use oral temozolomide, a DNA methylating agent that penetrates the CNS and is well tolerated.

Our study has limitations which may have falsely raised or lowered the true incidence of LMD. A higher rate might reflect our longstanding interest in this disease or tendency to investigate with neuro-imaging and lumbar puncture seemingly minor symptoms or signs. Conversely, the incidence might be spuriously low because craniospinal axis MRI/routine CSF examination were not routinely performed in all oligodendroglial tumor patients. We are also limited in the conclusions we can draw because of the very small number of patients. Therefore only the strongest risks of LMD were identified and trends we observed (e.g. the association of $1 p$ and $19 q$ co-deletion) may be confirmed in larger studies.

Finally, this study is also limited to classical clinical and pathological variables gleaned from cancer registry data and chart review. Central pathology review was not performed for our study, and the classification of the tumors might have varied between pathologists. Furthermore, diagnostic practices may have changed over the course of the study period that could impact the classification of tumors as pure oligodendroglioma or mixed oligodendrogliomas. We were unable to evaluate the association of ventricle opening during surgery to the development of LMD. Aside from some data on $1 p$ and $19 q$ status, our study does not further interrogate genomic/molecular factors that could either influence the classification of oligodendroglial tumors or contribute to the risk of LMD. We are now using genomic technologies in archival formalinfixed paraffin-embedded tumor samples in patients from this study to determine if we can identify genomic signatures associated with this rare complication.

Acknowledgments We thank the Families of Clark H. Smith and Kathleen Lorette for their generous support of Neuro-oncology. JGC holds the Alberta Cancer Foundation Chair in Brain Tumor Research. JC holds a Clinician Investigator Award from Alberta Innovates Health Solutions.

Open Access This article is distributed under the terms of the Creative Commons Attribution Noncommercial License which permits any noncommercial use, distribution, and reproduction in any medium, provided the original author(s) and source are credited.

\section{References}

1. Engelhard HH, Stelea A, Mundt A (2003) Oligodendroglioma and anaplastic oligodendroglioma: clinical features, treatment and prognosis. Surg Neurol 60:443-456

2. Bromberg J, Van den Bent MJ (2009) Oligodendrogliomas: molecular biology and treatment. The Oncologist 14:155-163 
3. Cairncross G, Berkey B, Shaw E et al (2006) Phase III trial of chemotherapy plus radiotherapy compared with radiotherapy alone for pure and mixed anaplastic oligodendroglioma: Intergroup Radiation Therapy Oncology Group Trial 9402. J Clin Oncol 24:2707-2714

4. van den Bent MJ, Carpentier AF, Brandes AA et al (2006) Adjuvant procarbazine, lomustine, and vincristine improves progression-free survival but not overall survival in newly diagnosed anaplastic oligodendrogliomas and oligoastrocytomas: a randomized European Organization for Research and Treatment of Cancer phase III trial. J Clin Oncol 24:2715-2722

5. Jenkins RB, Blair H, Ballman KV et al (2006) A t(1;19)(q10;p10) mediates the combined deletions of $1 p$ and $19 q$ and predicts a better prognosis of patients with oligodendroglioma. Cancer Res 66:9852-9861

6. Cairncross JG, Ueki K, Zlatescu MC et al (1998) Specific genetic predictors of chemotherapeutic response and survival in patients with anaplastic oligodendrogliomas. J Natl Cancer Inst 90: 1473-1479

7. Bauman GS, Ino Y, Ueki K et al (2000) Allelic loss of chromosome $1 \mathrm{p}$ and radiotherapy plus chemotherapy in patients with oligodendrogliomas. Int J Radiat Oncol Biol Phys 48:825-830
8. Ino Y, Betensky RA, Zlatescu MC, Sasaki H et al (2001) Molecular subtypes of anaplastic oligodendroglioma: implications for patient management at diagnosis. Clin Cancer Res $7: 839-845$

9. Roldán G, Scott J, George D, Parney I, Easaw J, Cairncross G, Forsyth P, Yan E (2008) Leptomeningeal disease from oligodendroglioma: clinical and molecular analysis. Can J Neurol Sci 35:204-209

10. Nielsen MS, Christensen HC, Kosteljanetz M, Johansen C (2009) Incidence of an survival from oligodendroglioma in Denmark, 1943-2002. Neuro Oncol 11:311-317

11. Ohgaki H, Kleihues P (2005) Population-based studies on incidence, survival rates, and genetic alterations in astrocytic and oligodendroglial gliomas. J Neuropathol Exp Neurol 64:479-489

12. Oechsle K, Lange-Brock V, Kruell A, Bokemeyer C, de Wit M (2010) Prognostic factors and treatment options in patients with leptomeningeal metastases of different primary tumors: a retrospective analysis. J Cancer Res Clin Oncol 136:1729-1735

13. Clarke JL, Perez HR, Jacks LM, Panageas KS, Deangelis LM (2010) Leptomeningeal metastases in the MRI era. Neurology 74:1449-1454 